\title{
A Visible-Light-Induced Sandwich-Type Photocell Using Dye-Containing Polymer Coating
}

\author{
Takeo SHIMIDZU* and Tomokazu IYODA \\ Division of Molecular Engineering, Graduate School of Engineering, \\ Kyoto University, Sakyo-ku, Kyoto 606, Japan
}

(Received June 13, 1984)

\begin{abstract}
A thin and flexible sandwich-type photocell with visible-light illumination is presented. The photocell is prepared by a dye-containing polymer coating on a semiconductordeposited film-type electrode. The advantages in applying a polymer coated electrode to a visiblelight-induced photocell with high efficiency are pointed out.

KEY WORDS Thin and Flexible Sandwich-Type Photocell / Visible-Light / Polymer Coating / Semiconductor-Deposited Film-Type Electrode /
\end{abstract}

Polymer coatings have recently become of great interest as a powerful method for modifying electrode surface properties. The advantages offered by these preparations over directly chemically bound monolayer and adsorption layer include ease of preparation, stability, complete surface coverage in high density, and a wide range of application. Among a wide variety of functions exhibited by these polymer coated electrodes, application to dye-sensitization of semiconductor electrodes is attractive in light-energy harvesting systems. ${ }^{1}$ Much investigation has carried out on soaking semiconductor electrodes in aqueous dye-containing solution. ${ }^{2,3}$ Only a slight amount of the dye in the vicinity of the electrode participates in the effective electron transfer responsible for photocurrent generation. ${ }^{4}$ Therefore, dye-sensitization which shifts the photoactions of a semiconductor electrode to visible-light region has the disadvantage of low quantum yield in photocurrent generation. Waste and low efficiency can be overcome by a dye-containing polymer coating of a semiconductor electrode in high density and close contact.

In this paper, a thin and flexible sandwich- type photocell driven by visible-light is presented, using a semiconductor-deposited filmtype electrode and a dye-containing polymer coating method. The photocell has high utility in view of its convenience of preparation, high efficiency, flexibility, and large potential for application to other systems.

\section{EXPERIMENTAL}

Figure 1 shows the structure of the present sandwich-type photocell consisting of a indium-tin oxide (ITO)-coated film electrode, an Au-coated film electrode, and a slurry electrolyte layer containing a sensitizer, a supporting polymer, and a supporting electrolyte. The transparent electrically conductive films, ${ }^{5}$ CELEC-K-EC and CELEC-G-4FX, were used as the electrode in the photocell. Neither etching nor pretreatment of the electrode was done prior to use. Ohmic contact was made with indium (Nakarai Chem.). The slurry electrolyte layer was coated on one of the electrodes over a $20 \mathrm{~mm} \times 30 \mathrm{~mm}$ of area and the sandwich-type photocell was built up by placing the other electrode on the electrode coated with the slurry electro- 


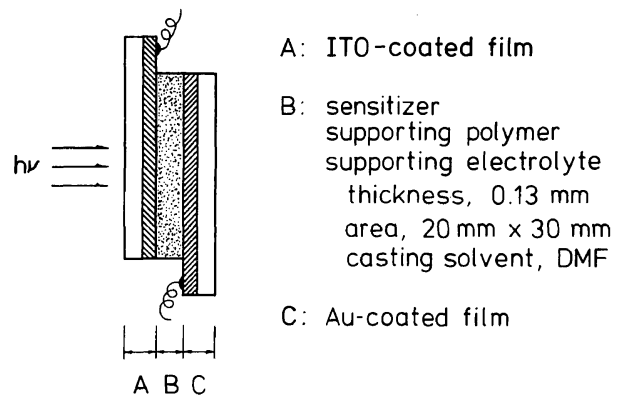

Figure 1. The structure of the present sandwich-type photocell.

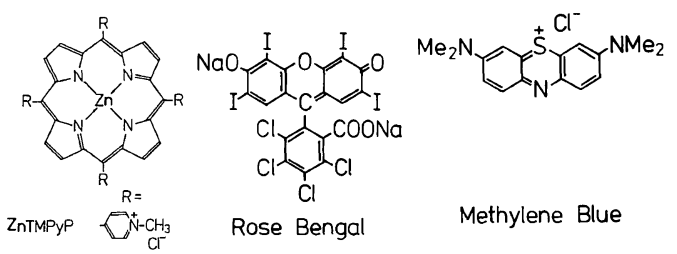

Figure 2. The structures and abbreviations of the sensitizers used.

lyte layer. The casting solution was made from a $0.5 \mathrm{~g} \cdot \mathrm{ml}^{-1} \mathrm{~N}, N$-dimethyl formamide (DMF) solution of a supporting polymer containing $0.1 \mathrm{~mol} \cdot 1^{-1}$ of a supporting electrolyte and a sensitizer. $\mathrm{NaClO}_{4}$ and tetrabutylammonium perchlorate (TBAP) (Nakarai Chem. and Merck) were used as supporting electrolytes. Supporting polymers were polyacrylonitrile, poly(acrylic acid), poly(vinylpyridine), polystyrene-4-sulfonic acid, and poly(vinyl alcohol). The thickness of the slurry electrolyte layer was $0.13 \mathrm{~mm}$. The structures and abbreviations of the sensitizers are shown in Figure 2.

Illumination was carried out with a $500 \mathrm{~W}$ Xe lamp through a grating spectroscope (JASCO, CT-25N) where the monochromatic light was $10^{-10}-10^{-9}$ einstein $\cdot \mathrm{s}^{-1}$ of the incident light intensity and $\pm 3 \mathrm{~nm}$ of the resolution. The incident light intensity was controlled by series of T.N.D. filters (Toshiba), and measured with a Reinecke's salt chemical actinometry. ${ }^{6}$ All experiments were carried out at room temperature. The anodic photocur-

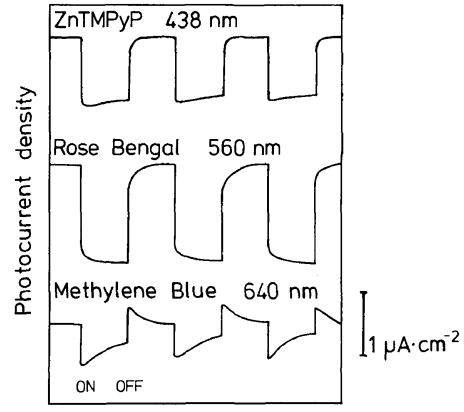

Figure 3. The anodic photocurrents in some dyesensitized sandwich-type photocells.

rent under short-circuit conditions was recorded on a HM-101 zero-shunt ammeter (Hokuto Denko). Visible absorption spectra of the sandwich-type photocell was recorded on a SM-401 spectrophotometer with the reference of the electrolyte layer free cell.

\section{RESULTS AND DISCUSSION}

Monochromatic light illumination $(2.5 \times$ $10^{-9}$ einstein $\cdot \mathrm{s}^{-1}$ ) responsible for the maximum absorption band of the adopted dye resulted in effective anodic photocurrent (in the direction from the electrolytic layer to the light-side electrode) in some dye-sensitized sandwich-type photocells. Figure 3 shows the photoresponses of the anodic current density $\left(J_{\text {sc }}\right)$ under short-circuit conditions. About $0.1 \mu \mathrm{A} \cdot \mathrm{cm}^{-2}$ of the anodic photocurrent density was observed in each photocell, whose photoresponse and reversibility were good toward the illumination. Table I shows the results of vital blank experiment in the present sandwich-type photocells. No photocurrent was observed when a dye, a supporting electrolyte, and a supporting polymer were lacking. Note that the ITO-coated film-type electrode in the light side plays a significant role in photocurrent generation. Only when a ITO film was used in the light-side electrode regardless of the dark-side electrode, could an effective photocurrent be observed. This observation showed that a Schottky-type potential 
Visible-Light-Induced Sandwich-Type Photocell

Table I. Photocurrents of various sandwich-type photocells consisting of film-type electrodes and sensitizer-containing polymer membranes $^{\mathbf{a}}$

\begin{tabular}{rcccccc}
\hline $\begin{array}{c}\text { Run. } \\
\text { No. }\end{array}$ & $\begin{array}{c}\text { Light-side } \\
\text { electrode }\end{array}$ & Sensitizer & $\begin{array}{c}\text { Supporting } \\
\text { electrolyte }\end{array}$ & $\begin{array}{c}\text { Supporting } \\
\text { polymer }\end{array}$ & $\begin{array}{c}\text { Dark-side } \\
\text { electrode }\end{array}$ & $\begin{array}{c}\text { Photocurrent }\left(\mu \mathrm{A} \cdot \mathrm{cm}^{-2}\right) / \\
\lambda \text { of incident light }\end{array}$ \\
\hline 1 & ITO & $3.3 \mathrm{mM} \mathrm{ZnTMPyP}$ & TBAP & AN & Au & $1.1 \times 10^{-1}(438 \mathrm{~nm})$ \\
2 & ITO & None & TBAP & AN & Au & $0^{*}$ \\
3 & ITO & $3.3 \mathrm{mM} \mathrm{ZnTMPyP}$ & None & AN & Au & $0^{*}$ \\
4 & ITO & $3.3 \mathrm{mM}$ ZnTMPyP & TBAP & AN & ITO & $1.1 \times 10^{-1}(438 \mathrm{~nm})$ \\
5 & Au & $3.3 \mathrm{mM}$ ZnTMPyP & TBAP & AN & ITO & $0^{*}$ \\
6 & Au & $3.3 \mathrm{mM}$ ZnTMPyP & TBAP & AN & Au & $0^{*}$ \\
7 & ITO & $3.3 \mathrm{mM} \mathrm{ZnTMPyP}$ & NaClO & AN & Au & $1.1 \times 10^{-1}(438 \mathrm{~nm})$ \\
8 & ITO & $3.3 \mathrm{mM}$ ZnTMPyP & TBAP & PVP & Au & $1.0 \times 10^{-1}(438 \mathrm{~nm})$ \\
9 & ITO & $3.3 \mathrm{mM} \mathrm{ZnTMPyP}$ & TBAP & PAA & Au & $1.1 \times 10^{-1}(438 \mathrm{~nm})$ \\
10 & ITO & $5.9 \mathrm{mM} \mathrm{RB}$ & TBAP & AN & Au & $1.6 \times 10^{-1}(560 \mathrm{~nm})$ \\
11 & ITO & $16.0 \mathrm{mM} \mathrm{MB}$ & TBAP & AN & Au & $7.5 \times 10^{-2}(640 \mathrm{~nm})$ \\
\hline
\end{tabular}

a TBAP, AN, PVP, PAA, RB, and MB are tetrabutylammonium perchlorate, polyacrylonitrile, poly(vinylpyridine), poly(acrylic acid), rose bengal, and methylene blue, respectively.

* No photocurrents were observed at any wavelength of incident light.

barrier formed at the interface between an ITO film electrode and a electrolytic layer contributed largely to the electron injection process from the dye in the excited state to the lightside ITO film electrode (Scheme 1).

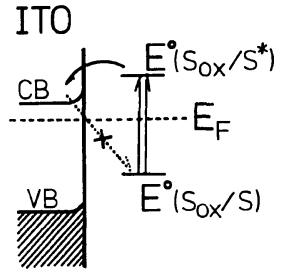

1,2

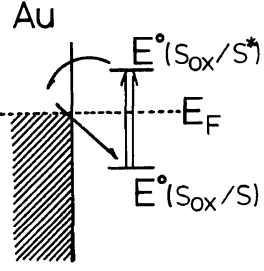

3,4
Scheme 1. The electron injection process from the excited dye to the ITO electrode or Au electrode.

Figures 4 and 5 show the dependences of the photocurrent at $438 \mathrm{~nm}$ in ZnTMPyPsensitized sandwich-type photocell on the light intensity (up to $6.0 \times 10^{-9}$ einstein $\cdot \mathrm{s}^{-1}$ ) and on the concentration of ZnTMPyP (up to $2.0 \times 10^{-2} \mathrm{~mol} \cdot \mathrm{l}^{-1}$ ), respectively. There are good linear relationships in Figures 4 and 5. Figure 6 shows the visible absorption spectrum of the photocell. The maximum absorption band at $438 \mathrm{~nm}$ in the photocell was

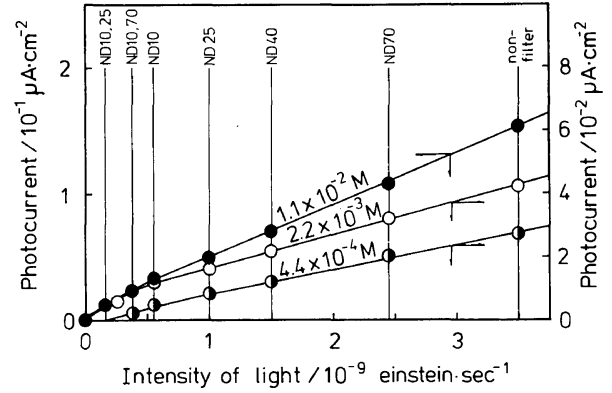

Figure 4. The dependence of photocurrent on the incident light intensity in the ZnTMPyP-sensitized sandwich-type photocell. [ZnTMPyP] $=1.1 \times 10^{-2} \mathrm{~mol}$ $1^{-1}(\bigcirc), 2.2 \times 10^{-3} \mathrm{moll}^{-1}(\bigcirc)$, and $4.4 \times 10^{-4} \mathrm{~mol}$ $1^{-1}(\mathbf{O})$.

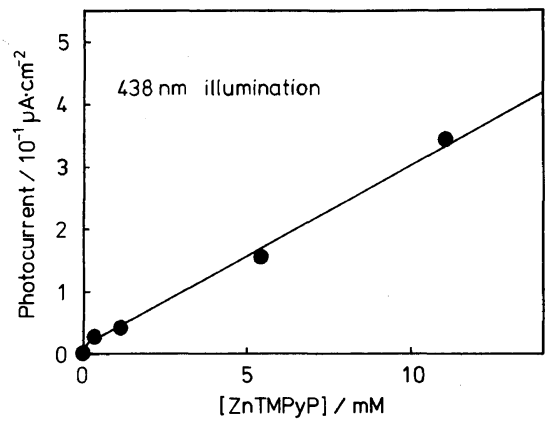

Figure 5. The dependence of photocurrent on the concentration of ZnTMPyP in the slurry electrolyte layer of the ZnTMPyP-sensitized sandwich-type photocell. 


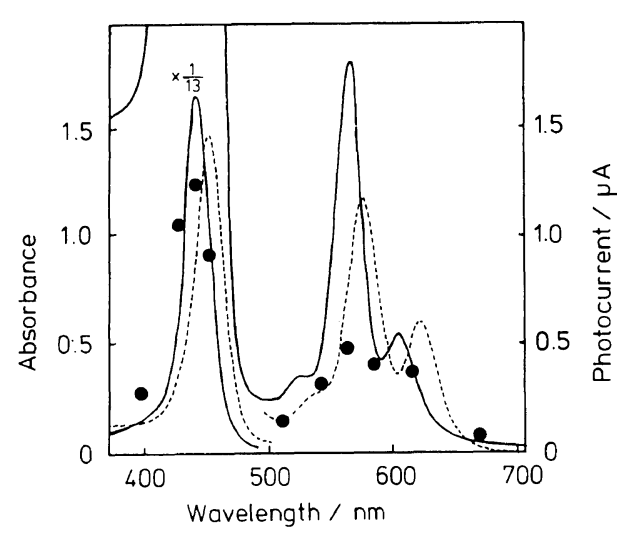

Figure 6. Visible absorption spectra of the photocell (-) and ZnTMPyP in DMF (-----), and the action spectrum of the photocurrent (O).

blue-shifted by $10 \mathrm{~nm}$ from that of ZnTMPyP in DMF, independent of the concentration of ZnTMPyP. This shift is explained by the polarity in the polymer matrices and not by the self-aggregation of ZnTMPyP. The action spectrum of the anodic photocurrent is also shown in Figure 6 under conditions satisfying the linear relationships in Figures 4 and 5. The agreement of the action spectrum with that of the visible absorption spectrum suggests that ZnTMPyP in polymer matrices functions as a sensitizer injecting an electron into the lightside ITO film electrode. The apparent currrent quantum yield was estimated to be $3.76 \%$ at $438 \pm 3 \mathrm{~nm},{ }^{7}$ when $[\mathrm{ZnTMPyP}]=1.1 \times 10^{-2}$ $\mathrm{mol} \cdot 1^{-1}$. This value is much larger than that $(<0.01 \%)$ in the case of only impregnation of the film electrode in a ZnTMPyP-containing aqueous solution. This apparent quantum yield depended linearly on the concentration of ZnTMPyP, based on the relationship in Figure 5. Saturation in the yield was observed when $[\mathrm{ZnTMPyP}]>2.0 \times 10^{-2} \mathrm{~mol} \cdot \mathrm{1}^{-1}$. In this case, only ZnTMPyP in the thin layer near the light-side ITO electrode could participate in the effective electron transfer to the ITO electrode owing to high absorbance of the cell. High efficiency is acquired in the present dyecontaining polymer coating method which has

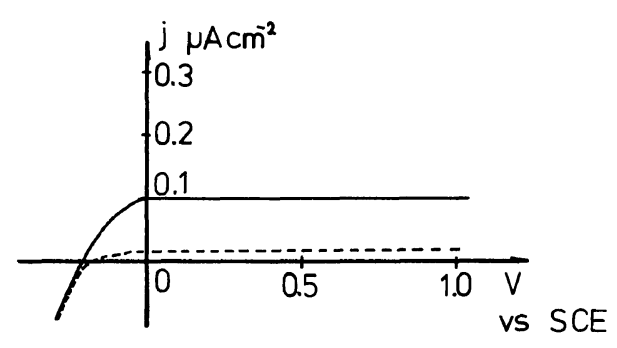

Figure 7. The current-potential curve of the ZnTMPyP-coated ITO film electrode in $0.1 \mathrm{~mol} \cdot 1^{-1}$ phosphate buffer ( $\mathrm{pH}$ 7). (-), under illumination; (-----), in the dark.

high density of the dye and close contact between the dye and the electrolyte layer to the electrode.

Under short-circuit conditions, the photocurrent was kept constant and no spectral change was observed. Conversely, a $33 \%$ of ZnTMPyP decomposed during $7.5 \mathrm{~h}$ illumination under an open-circuit condition. This condition perhaps results in a gradual irreversible side-reaction, predominant for the desired electron transfer to the ITO electrode in the light-side. Since there is no other redox couple except ZnTMPyP which mediates an electron transfer through the electrolyte layer, it is hard to transfer an electron from the counter electrode to the oxidized dye. In working photocell, the electron transfer through the electrolyte is considered to be mediated by an other ZnTMPyP, followed by electron exchange or diffusion of the oxidized ZnTMPyP. Although neither mechanism has been determined yet, good stability under the shortcircuit condition leads to the consideration that the electron transfer through the electrolyte layer may be very fast. The addition of $I_{2} / I^{-}\left(0.05 \mathrm{~mol} \cdot 1^{-1}\right)$ improved the durability of ZnTMPyP under the open-circuit condition.

Figure 7 shows the current-potential curve of the ZnTMPyP-coated ITO film electrode in $0.1 \mathrm{~mol} \cdot 1^{-1}$ phosphate buffer ( $\mathrm{pH} 7$ ). The current-potential curve was recorded at a $5 \mathrm{mV} \cdot \mathrm{s}^{-1}$ sweep rate of the electrode potential on a potentiostat when impregnating an elec- 
trolyte layer coated ITO electrode. Visible light illumination resulted in a photocurrent over the range of $>-2.0 \mathrm{~V} v s$. SCE. The behavior on the $\mathrm{i}-\mathrm{E}$ curve was similar to the case of the dye-sensitized semiconductor bulky electrode. ${ }^{2}$ This strongly suggests that the present photocurrent results from dyesensitization of the ITO film electrode with visible light illumination.

In conclusion, a dye-containing polymer coating of the semiconductor electrode such as the ITO film is available for semiconductor dye-sensitization due to the high density of the dye and close contact to the electrode. A thin and flexible sandwich-type photocell with high efficiency is demonstrated using an ITO-coated film and a dye-containing polymer electrolyte layer. The polymer coating method should find application in the preparation of chemically modified electrodes.

Acknowledgement. The authors are grateful to Daicel Chem. Ind., Ltd. for supplying the ITO-coated film and Au-coated film electrodes. This work was supported by Grand-inAid for Scientific Research from the Ministry of Education, Japan.

\section{REFERENCES AND NOTES}

1. W. J. Albery, A. W. Foulds, K. J. Hall, and A. R. Hillman, J. Electrochem. Soc., 127, 654 (1980).

2. H. Tsubomura, "Photoelectrochemistry and Energy Conversion," Tokyo Kagaku Dojin, Tokyo, 1980.

3. T. Shimidzu, T. Iyoda, K. Murai, N. Kanda, Y. Inoue, and K. Izaki, J. Electrochem. Soc., in press.

4. M. Matsumura, Y. Nomura, and H. Tsubomura, Bull. Chem. Soc. Jpn., 50, 2533 (1977).

5. A K-EC film has an electrically conductive thin layer made of indium tin oxide (ITO) with a 100 $500 \Omega \cdot$ square $^{-1}$ surface resistivity and a $80 \%$ visible light transmittance. A G-4FK film has a gold thin layer with a $4 \Omega \cdot$ square $^{-1}$ surface registivity and a $60 \%$ visible light transmittance. Both films are $1.25 \times 10^{-4} \mathrm{~m}$ in thickness. The supporting polymer film was made of polyester.

6. E. E. Wegner and A. W. Adamson, J. Am. Chem. Soc., 88, 394 (1966).

7. The apparent current quantum efficiency $(\eta)$ is given by the following equation.

$$
\eta=\frac{\text { (passed current in the external circuit) }}{\text { (moles of incident photons) }}
$$

Calculation was made assuming all the incident photons to be completely absorbed by the sensitizer and all the excited sensitizers to participate in electron injection into the electrode at $100 \%$ efficiency. 